\title{
RADIOSENSITIZATION OF CANCER STEM CELLS: TARGETING TGF $\beta$, NOTCH OR TELOMERASE TO IMPROVE TUMOR RESPONSE O RADIOTHERAPY
}

\author{
Ignacio Fernandez-Garcia \\ Department of Radiation Oncology, New York University School of Medicine, \\ 566 First Avenue, New York, New York, 10016, USA
}

Received 2012-05-31; Revised 2012-06-04; Accepted 2012-07-16

\begin{abstract}
Radiation resistant cancer stem cells are the main reason for treatment failure and tumor recurrence after cancer radiotherapy. Increasing biological evidences demonstrate that these cells possess the capacity to repair radiation induced DNA damage, protect themselves from radiation derived reactive oxygen species, survive and proliferate after several fractions of radiotherapy and finally, repopulate the heterogeneity of the tumor. Thus, targeting and eliminating these cells should be necessary to achieve cancer cure in radiotherapy. Three major approaches that specifically target radioresistant cancer stem cells have been recently investigated. First, inhibition of TGF $\beta$, a major mediator of the tissue response to radiation, has been shown to induce radiosensitization of cancer stem cells by targeting the DNA damage response mechanism. Second, by preventing Notch activation during fractionated radiotherapy, cancer stem cells were depleted from their ability to repopulate the tumor after radiation. Finally, telomerase activity inhibitors have shown to specifically decrease the cancer stem cell population after radiotherapy. In the present review, we evaluate these radiosentitizing approaches and their possible effects when combined with fractionated radiotherapy as they promise to be a powerful tool in the battle against this cancer.
\end{abstract}

Keywords: Cancer Stem Cells (CSC), Reactive Oxygen Species (ROS), Double Strand Breaks (DSB), Extra Cellular Matrix (ECM), Anticancer Treatments

\section{INTRODUCTION}

Between 1991 and 2007, cancer mortality rates in the US decreased $22.2 \%$ for men and $13.9 \%$ for women (Siegel et al., 2011), thanks in part to the development of early detection techniques, like the discovery of the prsotate-specific antigen (Toubert et al., 1996) and the improvements in appropriate treatment and palliative techniques including surgery, radiotherapy and chemotherapy. But despite these encouraging trends, cancer is still one of the leading causes of death in developed and developing countries and behavioral trends indicate an alarming increase in the exposure to risk factors, specially for low-and middle-income countries (McCormack and Boffetta, 2011). During 2008 , cancer alone was responsible for $13 \%$ of the total number of deaths worldwide, almost 7.6 millions and the incidence of new cases estimated for that year ascended to 12.6 millions (Boyle and Levin, 2008). More recently, 1.6 million new cases of cancer were estimated for 2011 in the US with a predicted mortality of almost 572,000 deaths (Siegel et al., 2011). In this context and even with the considerable reduction in mortality rates during the last twenty years, cancer is the leading cause of death among men and women younger than 85 in the US since 1999 (Landis et al., 1999).

It is also well established that despite the improvements achieved in the field of cancer therapy, the response to different treatments is still heterogeneous and unfortunately, the majority of the treated tumors maintain the capacity to reemerge after the treatment. This heterogeneity is not only observed in a population basis, but also in single tumors, animal models and even in cancer cells in culture and 
indicates that the response of single cancer cells to anticancer treatments is heterogeneous.

A hierarchical organization of cancer cells subpopulations has been known for more than fifty years, when serial transplantation experiments demonstrated differential metastatic potential within the cells of a given tumor (Southam and Brunschwig, 1961). In this context, one of the most accepted theories that explains cancer cells heterogeneity in their resistance to anticancer therapies resides in the existence of a sub-population of cells within the tumor with specific capabilities that make them particularly resistant to both chemo and radiotherapy (Eramo et al., 2006; Liu et al., 2006; Ghods et al., 2007; Kang and Kang, 2007; Mimeault et al., 2007; Ma et al., 2008). These cells possess the capacity to self renew and to generate the heterogeneous lineages of cancer cells that comprise the tumor (Clarke et al., 2006) and are known as Cancer Stem Cells (CSC) (Reya et al., 2001). However, the existence of CSCs is still controversial (Trott, 1994; Hill, 2006; Hill and Perris, 2007) mainly due to interpretation and nomenclature issues about what a stem cell truly is and to continuous discrepancies about what combination of surface biomarkers characterize and differentiate the CSC from other cancer cells (Baumann et al., 2008; Al-Assar et al. 2009; Alexander et al., 2009; McCord et al., 2009; Ropolo et al., 2009; Smith et al., 2011). In addition, few years ago (Mani et al., 2008) identified a connection between the stem cell signature and the epithelial to Mesenchymal Transition (EMT) program suggesting that differentiated cells may acquire stem capabilities. Some recent reports indicate the ability of single cancer cells to initiate tumors (Quintana et al., 2008) and this epithelial plasticity, although controversial since the EMT signature in differentiated cells differs from that executed in cancer cells when acquiring metastatic potential (reviewed in
(Zeisberg and Neilson, 2009), has been suggested as a mechanism to initiate tumors for non cancer stem cells.

Is for all of those controversial issues that, for the purpose of this review, we will not get into the existence of single deterministic stem cells in the tumor or the plasticity capabilities of all cancer cells to become cancer stem cells and we will just focus our intent in the variability of responses to cancer therapy that are observed as a result of the intrinsic tumor heterogeneity and how to overcome them with novel therapeutic approaches. To do that, we will consider the existence of a population of cells within the tumor that, after a radiotherapy has killed most of the tumor volume, remain almost unaffected and, more importantly, retain the capacity to regrow into a fully developed cancer (Pajonk et al., 2010) (Fig. 1) and in the shake of simplicity, we will retain the traditional and most common nomenclature that entitles these cells as CSCs.

Radiotherapy and cancer radioresistance: Radiotherapy is one of the least expensive cancer treatments and one of the most effective in terms of patient cure and overall survival (Dunscombe et al., 2007). In high-income countries, radiotherapy should be used for direct and palliative treatment in $52 \%$ of all new cancer cases (Delaney et al., 2005; Kimm et al., 2005). This numbers vary depending on the tumor type, but make radiotherapy particularly required for the treatment of several solid cancers like breast, lung and prostate (83, 76 and 60\% respectively) (Delaney et al., 2005). The principles in which radiation therapy bases its success and mechanisms of action when killing cancer cells were categorized by Whitners almost 40 years ago as the 4 R's of Radiobiology: Repair of DNA damage, Redistribution of cells in the cell cycle, Repopulation and Reoxygenation of hypoxic tumor areas (Withers, 1975).

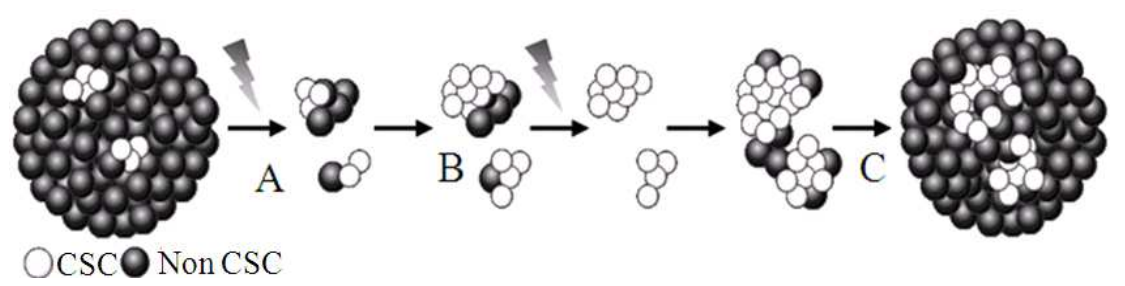

Fig. 1. Cancer recurrence after fractionated radiotherapy. (a) Radiation therapy induces DNA damage in cancer cells killing the majority of the tumor volume while acting as a selection mechanism for the intrinsically more resistant CSCs. (b) The surviving population of CSCs commence the repopulation of the tumor by reentering in cell cycle, proliferating and establishing an initial program of self renewal that increases their numbers. Further fractions of radiotherapy contribute to the selection of the CSC population and enrich the remaining tumor with symmetrically dividing CSC. (c) The increased population of CSC operates as seeds for proliferation, causing a rapid regrow of the tumor volume generally faster than the initial rate of the disease 
The most studied and well known effect of radiation and radiotherapy is the induction of double strand breaks in the DNA of the target cells. In that regards, high dose radiation, like the one employed for radiotherapy, induces direct ionization of the DNA causing a number of Double Strand Breaks (DSB) in the genome of a single cell directly proportional to the dose (Olive et al., 1991). In addition, as a physical property of ionizing radiation, highly Reactive Oxygen Species (ROS) are generated by the ionization of water molecules. Those induce local radical scavengers, such as glutathione that contribute as well to cause double strand breaks in the DNA (Mitchell and Russo, 1987). As a response to the damage, the cells activate a DNA Damage Response (DDR) mechanism capable to repair a certain amount of these breaks. The principle by which radiation is effective against cancer cells is because it generates enough damage to override the DDR mechanism and induce cell death.

However the activation of the DDR mechanism in cancer cells populations is heterogeneous as evidenced by differential survival of certain subpopulations after radiotherapy. For example, in gliobastoma a population of $\mathrm{CD}_{133^{+}}$putative cancer stem cells, was shown to be especially efficient in the repair of the radiation induced DSB as shown with the Alkaline Comet assay (Bao et al., 2006). Higher resistance to radiation was also found in breast cancer cell lines in mammosphere colony assays enriched for CD24 ${ }^{\text {low }}$ CD $44^{\text {high }}$ putative CSCs (Phillips et al., 2006), together with a more efficient repair of the DNA damage as evidenced by $\gamma \mathrm{H} 2 \mathrm{AX}$ foci (Diehn and Clarke, 2006; Phillips et al., 2006), a marker for the phosphorilation of the histone $\mathrm{H} 2 \mathrm{AX}$ after focal recognition of a DSB in the DNA (Olive, 2004). Pointing in the same direction, breast $\mathrm{LIN}^{-} \mathrm{CD} 24^{+} \mathrm{CD} 29^{+}$ mammosphere derived cells exhibited a distinct $\beta$ catenin and $\gamma \mathrm{H} 2 \mathrm{AX}$ activation patterns after radiation exposure, suggesting a possible role of the Wnt- $\beta$ catenin pathway in the DDR response to radiation induced DSBs (Chen et al., 2007; Woodward et al., 2007). In this context, inhibition of Chk1 phosphorilation, a downstream mediator of the $\gamma \mathrm{H} 2 \mathrm{AX}$ DDR pathway, has been shown to radiosensitize glioma and atypical teratoid/rhabdoid $\mathrm{CD}_{133^{+}}$cells (Bao et al., 2006; Chiou et al., 2008; Ropolo et al., 2009). In addition to the DDR activation, autophagy has been proposed as an alternative mechanism for the acquisition of radiation resistance employed by $\mathrm{CD} 133^{+}$cells and it has been shown that autophagy inhibition sensitized the cells to radiation reducing the sphere forming capacity of glioma CSCs (Lomonaco et al., 2009). Finally, described another mechanism of radiation resistance in breast cancer CSCs characterized by an antioxidant profile in which the CSCs showed increased expression of glutamate cystein ligase and glutathione synthetase. This translates into a more efficient scavenging of radiation induced ROS and results in less DSBs when compared to non CSCs irradiated at the same dose (Diehn et al., 2009).

Summarizing, CSCs exhibit an increased overall resistance to radiotherapy due to a more efficient activation of the DDR mechanism after radiation and to their capacity to minimize ROS induced DNA damage (Fig. 2). Fortunately, these characteristics also provide a new avenue for anticancer therapy and further evidences have demonstrated that, by specifically targeting them, there is an overall benefit in the tumors response to radiation.

TGF $\beta$ as a target for cancer stem cells Radiosensitization: One of the most interesting approaches to target the CSCs increased resistance to radiotherapy has been recently reported by three independent groups, in which breast cancer (Bouquet et al., 2011) and glioblastoma cell lines (Anido et al., 2010; Zhang et al., 2011) were radiosensitized by inhibition of Transforming Growth Factor $\beta$ (TGF $\beta$ ). Bouquet et al. (2011) reported in both colony forming assays and in vivo tumor control the inhibition of TGF $\beta$ with a small molecule or a neutralizing antibody increased the radiosensitivity of breast cancer. Interestingly, they showed that TGF $\beta$ inhibition reduces the efficiency of the DDR mechanism by specifically preventing the phosphorilation of ATM after radiation induced DNA damage (Bouquet et al., 2011). While the heterogeneity of the tumor populations response was not evaluated, (Anido et al., 2010) showed that inhibition of TGF $\beta$ specifically reduces the population of $\mathrm{CD}_{4} 4^{+} / \mathrm{Id} 1^{+}$ putative glioblastoma CSCs (Anido et al., 2010; Zhang et al., 2011) reported that glioblastoma CD133 ${ }^{+}$ cells in colony formation assays were specifically radiosensitize and suffered more DNA damage when the cultures were treated with the same inhibitors. Taken together, these studies indicate that the inhibition of TGF $\beta$ sensitize CSCs to radiotherapy by directly targeting their capacity to response to DNA damage (Fig. 2).

TGF $\beta$ is a family of cytokines involved in most biological processes including proliferation, migration, invasion, differentiation, angiogenesis, immune response and apoptosis (Moses et al., 2011). Just since its first role as an inhibitor of the mammary gland end buds development was identified by (Silberstein and Daniel, 1987) the controversy of its diametrically opposed roles arose as few months later, (Knabbe et al., 1987) showed that TGF $\beta$ was produced by the mammary gland to 
contribute to hormone resistance (Knabbe et al., 1987). Since then, TGF $\beta$ has been associated with tumor suppressor functions, controlling proliferation, apoptosis and instability, as well as with tumor promotion ones such as migration, invasion and plasticity.

The main regulator of TGF $\beta$ is the restrain of the active cytokine from the Latent complex (LTGF $\beta$ ) in which is secreted associated with the Latency Associated Peptide (LAP). This process of activation is the main controller for the bioactivity of TGF $\beta$. The LTGF $\beta$ complexes are secreted to the Extra Cellular Matrix (ECM) which serves as a reservoir (Flaumenhaft and Rifkin, 1992). These can be then locally activated through a wide variety of mechanisms including integrins, metalloproteinases, elastase, plasmin, thrombospondin (reviewed in (Moses et al., 2011)) and, more interestingly, ionizing radiation and ROS (Barcellos-Hoff and Dix, 1996). Upon activation, TGF $\beta$ binds to the type II TGF $\beta$ receptor (T $\beta$ IIR) promoting dimerization and transactivation of the type I TGF $\beta$ Receptor (T $\beta I R)$, triggering a downstream phosphorilation signaling of the SMAD transcription factors cascade (Derynck and Zhang, 2003; Massague, 2008). Activated SMADs then translocate from the cytoplasm to the nucleus where they regulate transcription of various target genes (reviewed in (Moses et al., 2011)) that translate into the different range of responses observed in cancer cells. The diametrically opposed response observed to TGF $\beta$ activation can be explained by a molecular balance of transcription factor C/EBP $\beta$ isoforms, LIP and LAP (Gomis et al., 2006) that controls a key program for the induction of c-myc and repression of p15. As a result, cancer cells become insensitive to proliferative and instability controls while acquire the malignant characteristics of invasion, motility and plasticity (Massague, 2008).

In the light of these roles of TGF $\beta$ in cancer establishment and progression it would be expected that, in addition to the DNA damage radiosentitizing effect described above, the inhibition of TGF $\beta$ can result in an overall benefit for radiotherapy as several capabilities of both CSCs and non CSCs will be compromised. For example, by inhibiting TGF $\beta$ it would be expected that the invasive and metastatic potential of the tumors will be reduced. Interestingly, that effect was shown by (Bouquet et al., 2011) as they reported a significant decrease in the number of lung metastasis in a xenograft model for breast cancer after treatment with radiation and TGF $\beta$ inhibitors (Bouquet et al., 2011). Such effect can be attributed to a direct inhibition of the EMT program which increases cancer cells motility and invasion through a TGF $\beta$ dependent activation of the transcription factors Snail and Twist (Zeisberg and Neilson, 2009).

Additionally, inhibition of TGF $\beta$ can provide a new interesting approach in radiosentitizing tumors and CSCs through its effect on reoxygenation. Hypoxic cancer cells show increased resistance to radiation (Thomlinson and Gray, 1955) especially when they are under intermittent hypoxic conditions (Zolzer and Streffer, 2002), which can be explained by the intermittent angiogenesis induced in the CSCs perivascular niches through the expression of Hypoxia-Inducible Factor $\alpha$ (HIF- $\alpha$ ) (Gustafsson et al., 2005; Pajonk et al., 2010). In contrast, persistent chronic hypoxia may increase radiosensitivity by decreasing RAD51-dependent DNA damage repair (Chan et al., 2008). A direct approach to induce chronic hypoxia is to prevent tumor angiogenesis. Interestingly, it has been shown that ionizing radiation may induce endothelial cell kill itself (Garcia-Barros et al., 2003; Imaizumi et al., 2010) and a synergistic effect between anti-angiogenic therapies and radiation has been observed (Seiwert and Cohen, 2008). TGF $\beta$ has been shown to stimulate angiogenesis through local transcriptional activation of Vascular Endothelial Growth Factor (VEGF) and Connective-Tissue Growth Factor (CTGF) (Sanchez-Elsner et al., 2001; Kang et al., 2003) and also by inducing endothelial cells to express monocyte chemoattractant Protein-1 (MCP-1) for the recruitment of vascular smooth muscle and mesenchymal cells toward the endothelium (Ma et al., 2007). Thus the power of inhibiting TGF $\beta$ in this scenario will reside in that, after radiotherapy, the surviving CSCs will be prevented for the possibility or reoxigenation and expansion, as angiogenesis will be blocked.

In essence, three major components of cancer malignancy can be targeted by inhibiting TGF $\beta$ in combination with radiotherapy. In addition to the specific radiosensitization effect caused in the CSCs, the tumor plasticity and angiogenesis capacity can be blocked resulting in an increased control of the tumor mass, essential for further targeting of the cancer cells in fractionated radiotherapy.

Targeting the cancer stem cell niche: The dependence of the CSCs to their local microenvironment is not only important for their response to reoxygenation and evidences accumulate showing that cancer cells are established in niches composed of several cell types that contribute to regulation and maintenance of the CSCs pool (Scadden, 2006; Gilbertson and Rich, 2007; Hambardzumyan et al., 2008). Is from these niches where, after radiotherapy has killed most of the tumor 
volume, radioresistant cancer stem cells exit the quiescent state in which they reside and initiate the repopulation of the tumor (Fig. 1).

Different cell cycle stages are characterized by different resistance to radiation, being cells in mitosis the most sensitive ones (Pawlik and Keyomarsi, 2004). Although there is only direct evidence from hematopoietic stem cells (Hoey et al., 2009; Korkaya and Wicha, 2009), CSCs are believed to exist in a quiescent G0 state in their niches and they become recruited to enter in cell cycle in order to initiate proliferation and repopulation of the tumor after radiotherapy. The Notch pathway has been identified as one of the molecular mechanisms implicated in this transition (Wu et al., 2007; Campa et al., 2008). It has been described that multiple radiation fractions promote Notch activation above the levels observed for single doses (Phillips et al., 2006). This is concomitant with an increase in the number of cycling CSCs (Vlashi et al., 2009) being recruited from their niches to repopulate the tumor and opens a new window for radiosensitization.

The repopulation capacity of the cancer stem cells, recruited from their perivascular niches to actively proliferate again, is the main reason for the failure of radiation therapy (Withers et al., 1988; Bese et al., 2005). This imply that CSCs in radiation treated cancers not only have to exit their quiescent stage, but also initiate a new program of symmetric and asymmetric divisions to establish the tumor heterogeneity, usually with faster growth rate than that from non treated tumors. This is done through an increased initial frequency of symmetric divisions that leads to a higher number of CSCs in the niches early after radiation, which later on will switch to asymmetric divisions to generate the progeny of heterogeneous tumor cells. Interestingly, the mechanism that orchestrate the decision between symmetric and asymmetric divisions in both normal tissue stem cells and in CSCs, employs the Notch, Wnt and hedgehog pathways (Phillips et al., 2006; Xu et al., 2008; Bisson and Prowse, 2009). In that regards several reports have shown activation of the Notch and Wnt pathways after radiation (Phillips et al., 2006; Woodward et al., 2007; Scharpfenecker et al., 2009).

Activation of the Notch pathway requires cell to cell contacts, where binding between the Notch receptor and ligands of the Delta or Jagged family occur. After binding, the Notch receptor is divided and the extracellular part is internalized into the ligand cell, while the remaining Notch is cleaved in the receptor cell by $\gamma$-secretase. This cleavage induces the release of the Notch receptor intracellular domain which translocates into the nucleus and binds to CBF-1, transforming this factor from transcriptional repressor to activator. As a result, several products are expressed to promote progression into the S-phase (Weinmaster and Kopan, 2006), recruitment into cell cycle (Campy) and maintenance of the stem cell phenotype (Wu et al., 2007) (Fig. 3).

Thus, the activation of the Notch pathway in CSCs can be targeted during fractionation radiotherapy to prevent the repopulation of the tumor after each fraction of radiation. As a prove of principle, the potential benefit of inhibiting Notch has been demonstrated by knockdown of Notch1 or Notch2 and ex vivo irradiation of glioma cells before injection, which resulted into extended tumor latency more than either treatment alone (Wang et al., 2010). In a recent report (Liu et al., 2011) showed that Notch inhibition both by $\gamma$-secretase inhibition and by delta ligand specific blockade resulted in a synergistic tumor growth delay when combined with radiotherapy in colorectal carcinoma and head and neck cancer cells (Liu et al., 2011). Interestingly, the group also reported a significant anti angiogenic effect of the delta ligand blockade when combined with radiotherapy, indicating that specific inhibition of the Notch pathway may interfere not only with the recruitment and repopulation capacity of the CSCs, but also with their ability to recruit endothelial cells to promote angiogenesis.

Telomerase targeting and radiotherapy: In addition to the window opened for the combination of radiotherapy with inhibitors for the Notch pathway, the existence of a CSCs niche that operates as a seed for tumor repopulation, uncovers a new possibility for radiosensitization in fractionated radiotherapy: Telomere targeting and the inhibition of telomerase activity.

The terminal regions of linear genomes are called Telomeres (Blackburn, 1984). They are formed by multiple tandem repetitions of a six nucleotide sequence that confers chromosomal stability and protects from irregular recombination, degradation and end to end fusions (Artandi and Attardi, 2005). A whole protein complex composed by six telomere-associated proteins called Shelterin, contributes to the stability of these terminal regions by creating a two-loops system that protects the telomeres from being recognized as a double strand break by the DNA damage response mechanism-conformation known as capped telomere-(De Lange, 2005).

Telomeres are the main cause for the "mitotic clock for aging" (Blasco, 2005). During the cell cycle, prior to the cell division at the end of the $\mathrm{S}$ phase when the DNA is finishing its replication, the polymerase complexes are 
unable to synthesize the end of the new strands of DNA, creating a gap in the 5' region (Olovnikov, 1973). This process will cause a progressive shortening of the telomeres in proliferating cells every time they divide (Harley, 1991) that will lead to the disruption of the protecting system enabled by the sheltering and the recognition of the telomeres as a DSB-uncapped telomeres-which will activate the cellular senescence pathway (Artandi and Attardi, 2005; De Lange, 2005). But this process can be bypassed by the expression of the telomerase protein complex. Telomerase is a DNA polymerase ribonucleoprotein complex Comprising an RNA subunit (TERC) and a Reverse Transcriptase component (TERT) that is express in normal stem cells and in embryonic tissues as a mechanism to prevent telomere attrition (Flores et al., 2006). The activation of the complex requires the interaction of its main subunits TERT and TERC with dyskerin 1 (Cohen et al., 2007), a protein for assembling and stabilizing the complex. Thus, the regulation of the telomerase is done through individual mechanisms that control the expression of its three major components and through the assembling process that binds them together (reviewed in (Flores et al., 2006; Collins, 2008).

In addition, the activity is mediated by the accessibility of the complex to the telomeres and as such, depends on the Shelterin complex conformation and its own regulation (De Lange, 2005; Seimiya et al., 2005). It is well known that telomerase is overexpressed in the majority of human cancers (Holt and Shay, 1999), where it acts as a mechanism to bypass telomere derived senescence and apoptosis. More importantly, ionizing radiation has been repeatedly shown to up regulate telomerase activity in several cancer cell lines in vitro (Finnon et al., 2000; Wang et al., 2000; Perez Mdel et al., 2002; Ram et al., 2009).

The differences between normal and cancer cells in terms of telomerase expression and telomere length provide a therapeutic opportunity for telomerase inhibition-based therapies. Cancer patients are less likely to develop resistance to telomerase-based therapies than to other cancer drugs and telomerase-based therapies are unlikely to cause tissue toxicity to normal non telomerase expressing cells (Harley, 2008). In addition, telomere dysfunction has been shown to enhance the radiosensitivity of cancer cells by decreasing the efficiency of the DDR mechanism in repairing the induced DNA (Wong et al., 2000). This characteristic has been employed to develop new agents that, by specifically targeting the telomeres conformation or the telomerase activity, increase radiosensitivity of cancer cells to radiation (Ayouaz et al., 2008; Wesbuer et al., 2010) (Fig. 4).

There are two different approaches for targeting telomerase activity: Direct enzyme inhibition and active telomerase immunotherapy. Different telomerase activity inhibitors have been proven to have anticancer properties in cell cultures in vitro as well as in animal models (Dikmen et al., 2005; Kelland, 2007). Furthermore, we have recently showed that inhibition of telomerase activity with small molecule inhibitors specifically enhances radiosensitivity of the AldH positive population of lung CSCs. We observed an increased tumor control in vivo after radiation and telomerase inhibition caused by the specific deletion of the AldH positive cells. Increased radiosensitivity of the AldH positive cells was also proven in vitro by sphere forming and colony survival assays (Serrano et al., 2011). As a different approach, cancer immunotherapies use synthetic TERT peptides to induce an immune response against cancer cells expressing TERT antigens. Recent results from phase I/II clinical trials have prove increased survival of patients with stage III non-small cell lung cancer when immunized with telomerase peptide vaccination in patients after having been treated with radiotherapy (Brunsvig et al., 2011). Although the specific response of the CSCs to the telomerase based immunotherapy was not evaluated, this results support the idea of a decrease in the proliferative capacity of the cancer cells after the combination of radiotherapy with the inhibition of telomerase. Finally, an example for direct telomere targeting for radiosensitization has been recently described by (Merle et al., 2011) in glioblastoma cells using a G-cuadruplex ligand that selectively binds to the spatial configuration of the telomeres causing instability and telomere uncapping. As a result, they showed a delay in the activation of the DDR mechanism by $\gamma \mathrm{H} 2 \mathrm{AX}$ and 53BP1 foci, resulting in enhanced radiosensitivity of GBM cells (Merle et al., 2011).

Given the specificity and selectivity of telomerase based therapies, the addition of a radiosensitization effect in the CSC population reinforce even more the potential in the combination of telomerase-telomere inhibition agents for controlling the tumor repopulation after radiotherapy. This way, the surviving cancer cells that remain after radiation will suffer from continuous telomere attrition under the telomerase inhibition treatment while proliferating to repopulate the tumor. This will finally cause telomere dysfunction and apoptosis, which contribute even further to control the tumor volume after several fractions of radiation. 


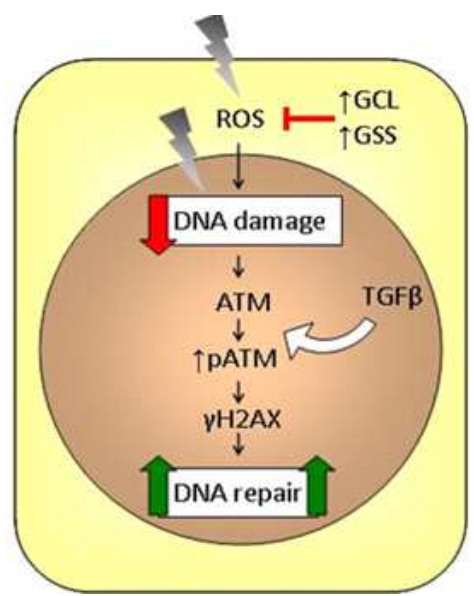

(a)

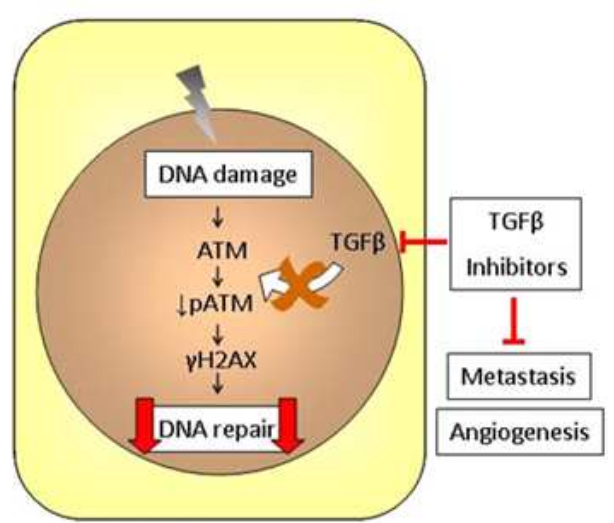

(b)

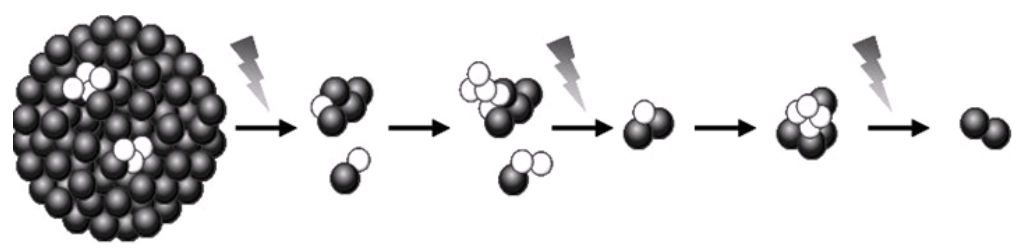

(c)

Fig. 2. TGF $\beta$ inhibition as a target for CSCs resistance to radiation induced DNA damage. (a) The increased radioresistance of CSCs is obtained by two intrinsic mechanisms: First, the DNA damage repair mechanism is generally activated more efficiently in CSCs than in non CSCs as evidenced by phosphorilation of ChK1 and ATM; Second, the overexpression of GCL and GSS facilitates the scavenging of radiation induced ROS, resulting in less DNA double strand breaks. (b) By targeting TGF $\beta$ with small molecule inhibitors or neutralizing antibodies cancer cells in general and CSCs in particular become more sensitive to radiation induced DNA damage due to a decrease in the phosphorilation of ATM and the consequent blockade of the DNA damage response mechanism. In addition, by inhibiting TGF $\beta$, several other cancer cells capabilities such as angiogenesis and metastasis can be compromised resulting in an overall benefit greater than radiosensitization itself. (c) A tumor populations model representation for the effects of TGF $\beta$ inhibition in combination with fractionated radiotherapy. Due to the radiosentitizing effect of inhibiting TGF $\beta$, the CSC subpopulation is no longer selected after radiotherapy, resulting in the eventual disappearance of the CSCs from the tumor after several fractions of radiation

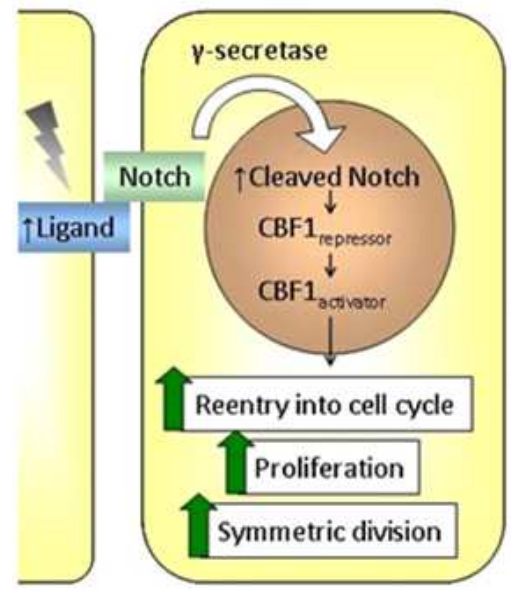

(a)

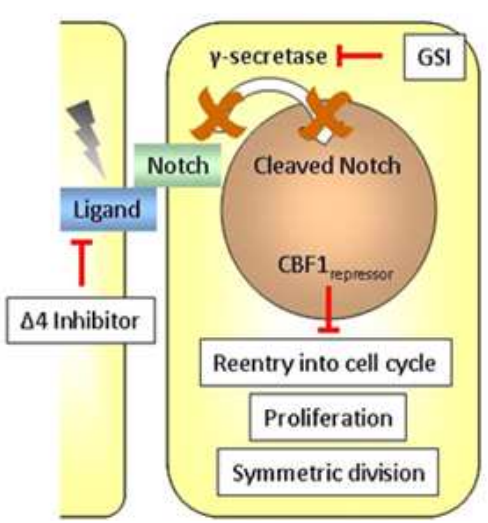

(b) 


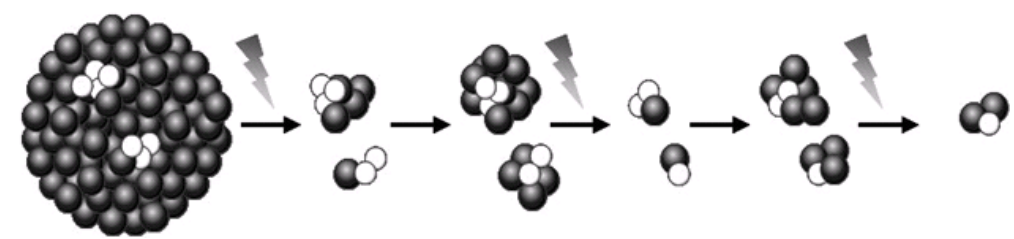

(c)

Fig. 3. Notch inhibition and the CSCs niche. (a) While radiotherapy kills most of the tumor cancer cells, the surviving CSCs remain quiescent in their niches where the Notch ligand is overexpressed after radiation. Notch is expressed in the membrane of CSCs in their niches and interaction between Notch and its ligand induces the internalization of the cleaved Notch fraction which then activates CBF-1 to promote reentry in to cell cycle, proliferation and symmetric division. This is translated in an overall increase of radioresistant and tumor initiating CSCs after each fraction of radiation. (b) Blocking the Notch ligand or specifically inhibiting the $\gamma$-secretases prevents cleaved Notch from binding to CBF- 1 . Thus, CBF- 1 remains as a transcriptional repressor preventing the maintenance of the stem cell phenotype. (c) In a model of fractionated radiotherapy, inhibition of the Notch pathway may result in an increased tumor control after each fraction of radiation, as the capacity of CSCs to proliferate in their niches in being compromise

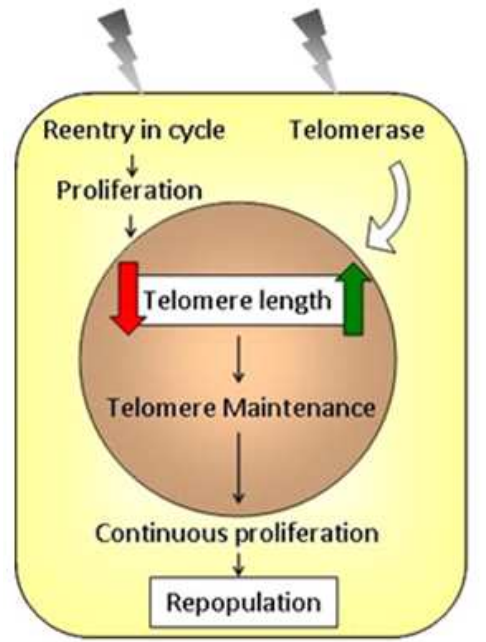

(a)

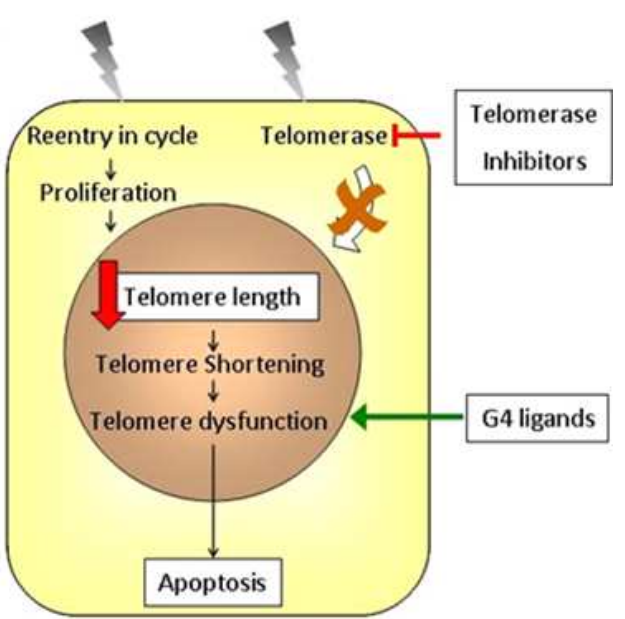

(b)

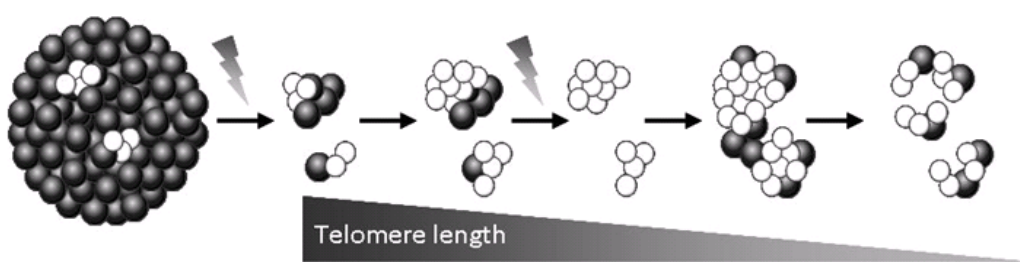

(c)

Fig. 4. Targeting the telomeres integrity for CSCs radiosensitization. (a) CSCs are stimulated to proliferate in their niches after radiotherapy has killed the radiation sensitive population of non CSCs. During the continuous cell divisions, overexpression of telomerase protects the telomeres from attrition and maintains a capped telomere conformation that facilitates further proliferation. (b) Inhibition of telomerase will cause continuous telomere shortening as the cells proliferate to repopulate the tumor after radiation. When the telomeres become critically short, the spatial protective conformation is compromised and the following uncapping induces apoptosis. The same effect can be triggered by specific agents that bind to the telomeric DNA G-cuadruplexes and destabilize the conformation to induce telomere dysfunction. (c) In a combination of radiotherapy with telomerase/telomere targeting agents, the surviving CSCs after radiation will suffer continuous telomere attrition and final apoptosis, as they proliferate in order to repopulate the tumor volume 


\section{CONCLUSION}

While the existence of a subpopulation of cells within the tumor capable to survive radiotherapy and cause cancer recurrence is worrisome, the discovery of the molecular mechanisms employed by these so called cancer stem cells to bypass therapy have revealed a new promising avenue for directed targeting that just recently has started to be exploited. The idea underlying these recent discoveries is that, by targeting the CSCs with specific inhibitors, the efficiency of conventional fractionated radiotherapy will be significantly improved. Furthermore, as these CSCs are supposed to be the true initiators of the tumor recurrence, they should be the main target for cancer treatment and radiotherapy.

We have shown three different approaches for specific CSCs radiosensitization, targeting each of them a different characteristic of the CSC biology. All three have resulted in very promising preliminary data in vitro and in animal models and some of them, like the telomerase inhibitor GRN163L and the telomerase peptide vaccine GV1001, are already in different phases of clinical trials. Hopefully, in the years to come more approaches and compounds will show the light and will prove their efficiency, the clinic by being able to eliminate CSCs and tumors when combined with radiotherapy.

\section{REFERENCES}

Al-Assar, O., R.J. Muschel, T.S. Mantoni, W.G. McKenna and T.B. Brunner, 2009. Radiation response of cancer stem-like cells from established human cell lines after sorting for surface markers. Int. J. Radiat. Oncol. Biol. Phys., 75: 1216-1225. DOI: 10.1016/j.ijrobp.2009.07.001

Alexander, C.M., J. Puchalski, K.S. Klos, N. Badders and L. Ailles et al., 2009. Separating stem cells by flow cytometry: Reducing variability for solid tissues. Cell Stem Cell, 5: 579-583.

DOI: $10.1016 /$ j.stem.2009.11.008

Anido, J., A. Saez-Borderias, A. Gonzalez-Junca, L. Rodon and G. Folch et al., 2010. TGF-beta receptor inhibitors target the CD44 (high)/Id1 (high) gliomainitiating cell population in human glioblastoma. Cancer Cell, 18: 655-668.

DOI: $10.1016 /$ j.ccr.2010.10.023

Artandi, S.E. and L.D. Attardi, 2005. Pathways connecting telomeres and p53 in senescence, apoptosis and cancer. Biochem. Biophys. Res. Commun., 331: 881-890.

DOI: $10.1016 /$ j.bbrc.2005.03.211
Ayouaz, A., C. Raynaud, C. Heride, D. Revaud and L. Sabatier, 2008. Telomeres: Hallmarks of radio sensitivity. Biochimie, 90: 60-72.

DOI: 10.1016/j.biochi.2007.09.011

Bao, S., Q. Wu, R.E. McLendon, Y. Hao and Q. Shi et al., 2006. Glioma stem cells promote radio resistance by preferential activation of the DNA damage response. Nature, 444: 756-760. DOI: 10.1038/nature05236

Barcellos-Hoff, M.H. and T.A. Dix, 1996. Redox-mediated activation of latent transforming growth factor- $\beta 1$. Mol. Endocrin., 10: 1077-1083. PMID: 8885242

Baumann, M., M. Krause and R. Hill, 2008. Exploring the role of cancer stem cells in radio resistance. Nature reviews. Cancer, 8: 545-554. DOI: 10.1038/nrc2419

Bese, N.S., P.A. Sut and A. Ober, 2005. The effect of treatment interruptions in the postoperative irradiation of breast cancer. Oncology, 69: 214-223. DOI: $10.1159 / 000087909$

Bisson, I. and D.M. Prowse, 2009. WNT signaling regulates self-renewal and differentiation of prostate cancer cells with stem cell characteristics. Cell Res., 19: 683-697. DOI: 10.1038/cr.2009.43

Blackburn, E.H., 1984. The molecular structure of centromeres and telomeres. Ann. Rev. Biochem., 53: 163-194. DOI: 10.1146/annurev.bi.53.070184.001115

Blasco, M.A., 2005. Telomeres and human disease: Ageing, cancer and beyond. Nat. Rev. Genet., 6: 611-622. DOI: 10.1038/nrg1656

Bouquet, F., A. Pal, K.A. Pilones, S. Demaria and B. Hann et al., 2011. TGFbeta1 inhibition increases the radiosensitivity of breast cancer cells in vitro and promotes tumor control by radiation in vivo. Clinical cancer research: An official J. Am. Associat. Cancer Res., 17: 6754-6765. DOI: 10.1158/1078-0432.CCR-11-0544

Boyle, P. and B. Levin, 2008. World cancer report 2008. World Health Organization.

Brunsvig, P.F., J.A. Kyte, C. Kersten, S. Sundstrom and M. Moller et al., 2011. Telomerase peptide vaccination in NSCLC: A phase II trial in stage III patients vaccinated after chemoradiotherapy and an 8-year update on a phase I/II trial. Clinical cancer research. Official J. Am. Associat. Cancer Res., 17: 6847-6857. DOI: 10.1158/1078-0432.CCR-11-1385

Campa, V.M., R. Gutierrez-Lanza, F. Cerignoli, R. DiazTrelles and B. Nelson et al., 2008. Notch activates cell cycle reentry and progression in quiescent cardiomyocytes. J. Cell Biol., 183: 129-141. DOI: $10.1083 /$ jcb.200806104 
Chan, N., M. Koritzinsky, H. Zhao, R. Bindra and P.M. Glazer et al., 2008. Chronic hypoxia decreases synthesis of homologous recombination proteins to offset chemoresistance and radioresistance. Cancer Res., 68: 605-614.

DOI: 10.1158/0008-5472.CAN-07-5472

Chen, M.S., W.A. Woodward, F. Behbod, S. Peddibhotla and M.P. Alfaro et al., 2007. Wnt/beta-catenin mediates radiation resistance of Sca1+ progenitors in an immortalized mammary gland cell line. J. Cell Sci., 120: 468-477. DOI: 10.1242/jcs.03348

Chiou, S.H., C.L. Kao, Y.W. Chen, C.S. Chien and S.C. Hung et al., 2008. Identification of CD133-positive radioresistant cells in atypical teratoid/rhabdoid tumor. PLoS One, 3: 2090-2090.

DOI: 10.1371/journal.pone.0002090

Clarke, M.F., J.E. Dick, P.B. Dirks, C.J. Eaves and C.H. Jamieson et al., 2006. Cancer stem cells-perspectives on current status and future directions: AACR workshop on cancer stem cells. Cancer Res., 66: 93399344. DOI: 10.1158/0008-5472.CAN-06-3126

Cohen, S.B., M.E. Graham, G.O. Lovrecz, N. Bache and P.J. Robinson et al., 2007. Protein composition of catalytically active human telomerase from immortal cells. Science, 315: 1850-1853.

DOI: $10.1126 /$ science. 1138596

Collins, K., 2008. Physiological assembly and activity of human telomerase complexes. Mechanisms Age. Dev., 129: 91-98. DOI: 10.1016/j.mad.2007.10.008

De Lange, T., 2005. Shelterin: The protein complex that shapes and safeguards human telomeres. Genes Dev., 19: 2100-2110. DOI: 10.1101/gad.1346005

Delaney, G., S. Jacob, C. Featherstone and M. Barton, 2005. The role of radiotherapy in cancer treatment: Estimating optimal utilization from a review of evidence-based clinical guidelines. Cancer, 104: 1129-1137. DOI: 10.1002/cncr.21324

Derynck, R. and Y.E. Zhang, 2003. Smad-dependent and Smad-independent pathways in TGF- $\beta$ family signalling. Nature, 425: 577-584.

DOI: 10.1038/nature02006

Diehn, M. and M.F. Clarke, 2006. Cancer stem cells and radiotherapy: New insights into tumor radio resistance. J. National Cancer Institute, 98: 1755-1757. DOI: 10.1093/jnci/djj505

Diehn, M., R.W. Cho, N.A. Lobo, T. Kalisky and M.J. Dorie et al., 2009. Association of reactive oxygen species levels and radioresistance in cancer stem cells. Nature, 458: 780-783. DOI: 10.1038/nature07733
Dikmen, Z.G., G.C. Gellert, S. Jackson, S. Gryaznov and R. Tressler et al., 2005. In vivo inhibition of lung cancer by GRN163L: A novel human telomerase inhibitor. Cancer Res., 65: 7866-7873.

DOI: 10.1158/0008-5472.CAN-05-1215

Dunscombe, P.B., S. Iftody, N. Ploquin, E.U. Ekaette and R.C. Lee, 2007. The equivalent uniform dose as a severity metric for radiation treatment incidents. Radiotherapy Oncol., 84: 64-66. DOI: $10.1016 /$ j.radonc.2007.05.024

Eramo, A., L. Ricci-Vitiani, A. Zeuner, R. Pallini and F. Lotti et al., 2006. Chemotherapy resistance of glioblastoma stem cells. Cell Death Differen., 13: 1238-1241. DOI: $10.1038 /$ sj.cdd.4401872

Finnon, P., A.R. Silver and S.D. Bouffler, 2000. Upregulation of telomerase activity by $\mathrm{X}$-irradiation in mouse leukaemia cells is independent of Tert, Terc, Tnks and Myc transcription. Carcinogenesis, 21: 573-578.

Flaumenhaft, R. and D.B. Rifkin, 1992. The extracellular regulation of growth factor action. Mol. Biol. Cell, 3: 1057-1065. PMID: 1421565

Flores, I., R. Benetti and M.A. Blasco, 2006. Telomerase regulation and stem cell behaviour. Curr. Opin. Cell Biol., 18: 254-260. DOI: S0955-0674(06)00046-9 [pii] 10.1016/j.ceb.2006.03.003

Garcia-Barros, M., F. Paris, C. Cordon-Cardo, D. Lyden and S. Rafii et al., 2003. Tumor response to radiotherapy regulated by endothelial cell apoptosis. Science, 300: 1155-1159. DOI: $10.1126 /$ science. 1082504

Ghods, A.J., D. Irvin, G. Liu, X. Yuan and I.R. Abdulkadir et al., 2007. Spheres isolated from 9L gliosarcoma rat cell line possess chemo resistant and aggressive cancer stem-like cells. Stem Cells, 25: 1645-1653. DOI: 10.1634/stemcells.2006-0624

Gilbertson, R.J. and J.N. Rich, 2007. Making a tumour's bed: Glioblastoma stem cells and the vascular niche. Nat. Rev. Cancer, 7: 733-736.

DOI: $10.1038 / \mathrm{nrc} 2246$

Gomis, R.R., C. Alarcon, C. Nadal, C. Van Poznak and J. Massague, 2006. C/EBPbeta at the core of the TGFbeta cytostatic response and its evasion in metastatic breast cancer cells. Cancer Cell, 10: 203214. DOI: S1535-6108(06)00247-9. DOI: $10.1016 /$ j.ccr.2006.07.019

Gustafsson, M.V., X. Zheng, T. Pereira, K. Gradin and S. Jin et al., 2005. Hypoxia requires notch signaling to maintain the undifferentiated cell state. Dev. Cell, 9: 617-628. DOI: 10.1016/j.devcel.2005.09.010 
Hambardzumyan, D., O.J. Becher, M.K. Rosenblum, P.P. Pandolfi and K. Manova-Todorova et al., 2008. PI3K pathway regulates survival of cancer stem cells residing in the perivascular niche following radiation in medulloblastoma in vivo. Genes Dev., 22: 436-448. DOI: $10.1101 /$ gad.1627008

Harley, C.B., 1991. Telomere loss: Mitotic clock or genetic time bomb. Mutat. Res. DNAg., 256: 271282. DOI: 10.1016/0921-8734(91)90018-7

Harley, C.B., 2008. Telomerase and cancer therapeutics. Nat. Rev. Cancer, 8: 167-179. DOI: $10.1038 / \mathrm{nrc} 2275$

Hill, R.P. and R. Perris, 2007. Destemming cancer stem cells. J. Nat. Cancer Institute, 99: 1435-1440. DOI: $10.1093 /$ jnci/djm136

Hill, R.P., 2006. Identifying cancer stem cells in solid tumors: Case not proven. Cancer Res., 66: 18911895. DOI: 10.1158/0008-5472.CAN-05-3450

Hoey, T., W.C. Yen, F. Axelrod, J. Basi and L. Donigian et al., 2009. DLL4 blockade inhibits tumor growth and reduces tumor-initiating cell frequency. Cell Stem Cell, 5: 168-177.

DOI: $10.1016 /$ j.stem.2009.05.019

Holt, S.E. and J.W. Shay, 1999. Role of telomerase in cellular proliferation and cancer. J. Cell Physiol., 180: 10-18. DOI: 10.1002/(SICI)10974652(199907)180:1<10::AID-JCP2>3.0.CO;2-D

Imaizumi, N., Y. Monnier, M. Hegi, R.O. Mirimanoff and C. Ruegg, 2010. Radiotherapy suppresses angiogenesis in mice through TGF-betaRI/ALK5dependent inhibition of endothelial cell sprouting. PLoS One, 5: 11084-11084.

DOI: 10.1371/journal.pone.0011084

Kang, Y., P.M. Siegel, W. Shu, M. Drobnjak and S.M. Kakonen et al., 2003. A multigenic program mediating breast cancer metastasis to bone. Cancer Cell, 3: 537549. DOI: 10.1016/S1535-6108(03)00132-6

Kang, M.K. and S.K. Kang, 2007. Tumorigenesis of chemotherapeutic drug-resistant cancer stem-like cells in brain glioma. Stem Cells Dev., 16: 837-847. DOI: $10.1089 / \mathrm{scd} .2007 .0006$

Kelland, L., 2007. Targeting the limitless replicative potential of cancer: The telomerase/telomere pathway. Clin. Cancer Res., 13: 4960-4963. DOI: 10.1158/1078-0432.CCR-07-0422

Kimm, S.Y., N.W. Glynn, E. Obarzanek, A.M. Kriska and S.R. Daniels et al., 2005. Relation between the changes in physical activity and body-mass index during adolescence: A multicentre longitudinal study. Lancet, 366: 301-307. DOI: $10.1016 / \mathrm{S} 0140-6736(05) 66837-7$
Knabbe, C., M.E. Lippman, L.M. Wakefield, K.C. Flanders and A. Dasid et al., 1987. Evidence that transforming growth factor- $\beta$ is a hormonally regulated negative growth factor in human breast cancer cells. Cell, 48: 417-428. DOI: 10.1016/0092-8674(87)90193-0

Korkaya, H. and M.S. Wicha, 2009. HER-2, notch and breast cancer stem cells: Targeting an axis of evil. Clin. Cancer Res., 15: 1845-1847.

DOI: 10.1158/1078-0432.CCR-08-3087

Landis, S.H., T. Murray, S. Bolden and P.A. Wingo, 1999. CA: Cancer J. Clin., 49: 8-31. DOI: $10.3322 /$ canjclin.49.1.8

Liu, G., X. Yuan, Z. Zeng, P. Tunici and H. Ng et al., 2006. Analysis of gene expression and chemoresistance of CD133+ cancer stem cells in glioblastoma. Molecular Cancer, 5: 67. DOI: 10.1186/1476-4598-5-67

Liu, S.K., S.A. Bham, E. Fokas, J. Beech and J. Im et al., 2011. Delta-like ligand 4-notch blockade and tumor radiation response. J. National Cancer Institute, 103: 1778-1798. DOI: 10.1093/jnci/djr419

Lomonaco, S.L., S. Finniss, C. Xiang, A. Decarvalho and F. Umansky et al., 2009. The induction of autophagy by gamma-radiation contributes to the radio resistance of glioma stem cells. J. Int. Du Cancer, 125: 717-722. DOI: 10.1002/ijc.24402

Ma, J., Q. Wang, T. Fei, J.D. Han and Y.G. Chen, 2007. MCP-1 mediates TGF-beta-induced angiogenesis by stimulating vascular smooth muscle cell migration. Blood, 109: 987-994. DOI: 10.1182/blood-2006-07-036400

Ma, S., T.K. Lee, B.J. Zheng, K.W. Chan and X.Y. Guan, 2008. CD133+ HCC cancer stem cells confer chemo resistance by preferential expression of the Akt/PKB survival pathway. Oncogene, 27: 1749-1758.

DOI: $10.1038 /$ sj.onc.1210811

Mani, S.A., W. Guo, M.J. Liao, E.N. Eaton and A. Ayyanan et al., 2008. The Epithelial-mesenchymal transition generates cells with properties of stem cells. Cell, 133: 704-715.

DOI: 10.1016/j.cell.2008.03.027

Massague, J., 2008. TGFbeta in Cancer. Cell, 134: 215230. DOI: 10.1016/j.cell.2008.07.001

McCord, A.M., M. Jamal, E.S. Williams, K. Camphausen and P.J. Tofilon, 2009. CD133+ glioblastoma stem-like cells are radiosensitive with a defective DNA damage response compared with established cell lines. Clinical Cancer Res., 15: 5145-5153. DOI: 10.1158/1078-0432.CCR-09-0263 
McCormack, V.A. and P. Boffetta, 2011. Today’s lifestyles, tomorrow's cancers: Trends in lifestyle risk factors for cancer in low-and middle-income countries. Ann. Oncol., 22: 2349-2357. DOI: 10.1093/annonc/mdq763

Merle, P., B. Evrard, A. Petitjean, J.M. Lehn and M.P. Teulade-Fichou et al., 2011. Telomere targeting with a new G4 ligand enhances radiation-induced killing of human glioblastoma cells. Molecular Cancer Therapeut., 10: 1784-1795. DOI: 10.1158/1535-7163.MCT-10-0664

Mimeault, M., R. Hauke, P.P. Mehta and S.K. Batra, 2007. Recent advances in cancer stem/progenitor cell research: Therapeutic implications for overcoming resistance to the most aggressive cancers. J. Cellular Molecular Med., 11: 981-1011. DOI: $10.1111 / \mathrm{j} .1582-4934.2007 .00088 . x$

Mitchell, J.B. and A. Russo, 1987. The role of glutathione in radiation and drug induced cytotoxicity. Br. J. Cancer Suppl., 8: 96-104.

PMID: 3307879

Moses, H. and M.H. Barcellos-Hoff, 2011. TGF-beta biology in mammary development and breast cancer. Cold Spring Harbor Perspectives Biol., 3: 003277-003277. DOI: 10.1101/cshperspect.a003277

Olive, P.L., 2004. Detection of DNA damage in individual cells by analysis of histone $\mathrm{H} 2 \mathrm{AX}$ phosphorylation. Methods Cell Biol., 75: 355-373. PMID: 15603433

Olive, P.L., D. Wlodek and J.P. Banath, 1991. DNA double-strand breaks measured in individual cells subjected to gel electrophoresis. Cancer Res., 51: 4671-4676.

Olovnikov, A.M., 1973. A theory of marginotomy. The incomplete copying of template margin in enzymic synthesis of polynucleotides and biological significance of the phenomenon. J. Theor. Biol., 41: 181-190. PMID: 4754905

Pajonk, F., E. Vlashi and W.H. McBride, 2010. Radiation resistance of cancer stem cells: The 4 R's of radiobiology revisited. Stem Cells, 28: 639-648. DOI: $10.1002 /$ stem.318

Pawlik, T.M. and K. Keyomarsi, 2004. Role of cell cycle in mediating sensitivity to radiotherapy. Int. J. Radiat. Oncol. Biol. Phys., 59: 928-942. DOI: 10.1016/j.ijrobp.2004.03.005

Perez Mdel, R., D. Dubner, S. Michelin, F. Leteurtre and E.D. Carosella et al., 2002. Radiation-induced upregulation of telomerase in KG1a cells is influenced by dose-rate and radiation quality. Int. J. Radiat. Biol., 78: 1175-1183. DOI: 10.1080/0955300021000013812
Phillips, T.M., W.H. McBride and F. Pajonk, 2006. The response of CD24 (-/low)/CD44+ breast cancerinitiating cells to radiation. J. Nat. Cancer Institute, 98: 1777-1785. DOI: 10.1093/jnci/djj495

Quintana, E., M. Shackleton, M.S. Sabel, D.R. Fullen and T.M. Johnson et al., 2008. Efficient tumour formation by single human melanoma cells. Nature, 456: 593-598. DOI: 10.1038/nature07567

Ram, R., O. Uziel, O. Eldan, E. Fenig and E. Beery et al., 2009. Ionizing radiation up-regulates telomerase activity in cancer cell lines by post-translational mechanism via ras/phosphatidylinositol 3-kinase/Akt pathway. Clinical Cancer Res., 15: 914-923. DOI: 10.1158/1078-0432.CCR-08-0792

Reya, T., S.J. Morrison, M.F. Clarke and I.L. Weissman, 2001. Stem cells, cancer and cancer stem cells. Nature, 414: 105-111. DOI: 10.1038/35102167

Ropolo, M., A. Daga, F. Griffero, M. Foresta and G. Casartelli et al., 2009. Comparative analysis of DNA repair in stem and nonstem glioma cell cultures. Molecular Cancer Res. MCR, 7: 383-392. DOI: 10.1158/1541-7786.MCR-08-0409

Sanchez-Elsner, T., L.M. Botella, B. Velasco, A. Corbi and L. Attisano et al., 2001. Synergistic cooperation between hypoxia and transforming growth factorbeta pathways on human vascular endothelial growth factor gene expression. J. Biol. Chem., 276: 38527-38535. DOI: 10.1074/jbc.M104536200

Scadden, D.T., 2006. The stem-cell niche as an entity of action. Nature, 441: 1075-1079.

DOI: 10.1038 /nature04957

Scharpfenecker, M., J.J. Kruse, D. Sprong, N.S. Russell and P. Ten Dijke et al., 2009. Ionizing radiation shifts the PAI-1/ID-1 balance and activates notch signaling in endothelial cells. Int. J. Radiat. Oncol. Biol. Phys., 73: 506-513.

DOI: 10.1016/j.ijrobp.2008.09.052

Seimiya, H., Y. Muramatsu, T. Ohishi and T. Tsuruo, 2005. Tankyrase 1 as a target for telomere-directed molecular cancer therapeutics. Cancer Cell, 7: 2537. DOI: 10.1016/j.ccr.2004.11.021

Seiwert, T.Y. and E.E. Cohen, 2008. Targeting angiogenesis in head and neck cancer. Seminars Oncol., 35: 274-285.

DOI: $10.1053 /$ j.seminoncol.2008.03.005

Serrano, D., A.M. Bleau, I. Fernandez-Garcia, T. Fernandez-Marcelo and P. Iniesta et al., 2011. Inhibition of telomerase activity preferentially targets aldehyde dehydrogenase-positive cancer stem-like cells in lung cancer. Molecular Cancer, 10: 96-96. DOI: 10.1186/1476-4598-10-96 
Siegel, R., E. Ward, O. Brawley and A. Jemal, 2011. Cancer statistics, 2011: The impact of eliminating socioeconomic and racial disparities on premature cancer deaths. CA: Cancer J. Clin., 61: 212-236. DOI: $10.3322 /$ caac. 20121

Silberstein, G.B. and C.W. Daniel, 1987. Reversible inhibition of mammary gland growth by transforming growth factor- $\beta$. Science, 237: 291-293. PMID: 3474783

Smith, B.H., L.S. Gazda, B.L. Conn, K. Jain and S. Asina et al., 2011. Three-dimensional culture of mouse renal carcinoma cells in agarose macrobeads selects for a subpopulation of cells with cancer stem cell or cancer progenitor properties. Cancer Res., 71: 716-724. DOI: 10.1158/0008-5472.CAN-10-2254

Southam, C.M. and A. Brunschwig, 1961. Quantitative studies of autotransplantation of human cancerpreliminary report. Cancer, 14: 971-978.

DOI: 10.1002/1097-0142(196109

Thomlinson, R.H. and L.H. Gray, 1955. The histological structure of some human lung cancers and the possible implications for radiotherapy. Br. J. Cancer, 9: 539-549. PMID: 13304213

Toubert, M.E., J. Guillet, M. Chiron, P. Meria and C. Role et al., 1996. Percentage of free serum prostatespecific antigen: A new tool in the early diagnosis of prostatic cancer. Eur. J. Cancer, 32A: 2088-2093. PMID: 9014750

Trott, K.R., 1994. Tumour stem cells: The biological concept and its application in cancer treatment. Radiol. Oncol., 30: 1-5. DOI: 10.1016/0167-8140(94)90002-7

Vlashi, E., K. Kim, C. Lagadec, L.D. Donna and J.T. McDonald et al., 2009. In vivo imaging, tracking and targeting of cancer stem cells. J. National Cancer Institute, 101: 350-359.

DOI: $10.1093 /$ jnci/djn509

Wang, J., T.P. Wakeman, J.D. Lathia, A.B. Hjelmeland and X.F. Wang et al., 2010. Notch promotes radioresistance of glioma stem cells. Stem Cells, 28: 17-28. DOI: 10.1002/stem.261

Wang, X., Y. Liu, L.S. Chow, S.C. Wong and G.S. Tsao et al., 2000. Regulation of telomerase activity by gamma-radiation in nasopharyngeal carcinoma cells. Anticancer Res., 20: 433-437. PMID: 10769692

Weinmaster, G. and R. Kopan, 2006. A garden of Notchly delights. Development, 133: 3277-3282. DOI: $10.1242 /$ dev.02515
Wesbuer, S., C. Lanvers-Kaminsky, I. Duran-Seuberth, T. Bolling and K.L. Schafer et al., 2010. Association of telomerase activity with radio-and chemosensitivity of neuroblastomas. Radiat. Oncol., 19: 5-66. DOI: 10.1186/1748-717X-5-66

Withers, H.R., 1975. The four R's of radiotherapy. Adv. Radiat. Biol. J. Lett. Adler Acad. Press, 5: 241-271.

Withers, H.R., B. Maciejewski, J.M. Taylor and A. Hliniak, 1988. Accelerated repopulation in head and neck cancer. Front. Radiat. Ther. Oncol., 22: 105-110. PMID: 3350350

Wong, K.K., S. Chang, S.R. Weiler, S. Ganesan and J. Chaudhuri et al., 2000. Telomere dysfunction impairs DNA repair and enhances sensitivity to ionizing radiation. Nat. Genet., 26: 85-88.

DOI: 10.1038/79232

Woodward, W.A., M.S. Chen, F. Behbod, M.P. Alfaro and T.A. Buchholz et al., 2007. WNT/beta-catenin mediates radiation resistance of mouse mammary progenitor cells. Proc. National Acad. Sci. United States Am., 104: 618-623. DOI: $10.1073 /$ pnas.0606599104

Wu, M., H.Y. Kwon, F. Rattis, J. Blum and C. Zhao et al., 2007. Imaging hematopoietic precursor division in real time. Cell Stem Cell, 1: 541-554.

DOI: 10.1016/j.stem.2007.08.009

Xu, Q., X. Yuan, G. Liu, K. L. Black and J. S. Yu, 2008. Hedgehog signaling regulates brain tumor-initiating cell proliferation and portends shorter survival for patients with PTEN-coexpressing glioblastomas. Stem Cells, 26: 3018-3026.

DOI: $10.1634 /$ stemcells.2008-0459

Zeisberg, M. and E.G. Neilson, 2009. Biomarkers for epithelial-mesenchymal transitions. J. Clin. Invest., 119: 1429-1437. PMID: 19487819

Zhang, M., T.W. Herion, C. Timke, N. Han and K. Hauser et al., 2011. Trimodal glioblastoma treatment consisting of concurrent radiotherapy, temozolomide and the novel TGF-beta receptor I kinase inhibitor LY2109761. Neoplasia, 13: 537-549. DOI: $10.1593 /$ neo. 11258

Zolzer, F. and C. Streffer, 2002. Increased radiosensitivity with chronic hypoxia in four human tumor cell lines. Int. J. Radiat. Oncol., Biol. Phys., 54: 910-920. 